\title{
ESTRUCTURA Y RELACIONES GENÉTICAS DE LA RAZA BOVINA SERRANA DE TERUEL CON RAZAS EXPLOTADAS EN ESPAÑA
}

\author{
STRUCTURE AND GENETIC RELATIONSHIPS BETWEEN SERRANA DE TERUEL \\ BREED AND OTHER CATTLE BREEDS REARED IN SPAIN
}

\author{
Sanz, A. ${ }^{1 *}$, Rodellar, C. ${ }^{1}$, Martín-Burriel, I. ${ }^{1}$, Sanz, A. ${ }^{2}$, Cons, C. ${ }^{1}$, Abril, F. ${ }^{3}$, Azor, P.J. ${ }^{4}$, \\ Piedrafita, J. ${ }^{5}$, Vijil, E. ${ }^{6}$ y Zaragoza, P. ${ }^{1}$
}

\begin{abstract}
${ }^{1}$ Laboratorio de Genética Bioquímica (LAGENBIO). Facultad de Veterinaria. Universidad de Zaragoza. Zaragoza. España. *arianne@unizar.es

${ }^{2}$ Unidad de Tecnología en Producción Animal. Centro de Investigación y Tecnología Agroalimentaria(CITA). Gobierno de Aragón. Zaragoza. España.

${ }^{3}$ Diputación Provincial de Teruel (ASERNA). Teruel. España.

${ }^{4}$ Departamento de Genética. Facultad de Veterinaria. Universidad de Córdoba. Córdoba. España.

${ }^{5}$ Departamento de Ciencia Animal y de los Alimentos. Facultad de Veterinaria. Universidad Autónoma de Barcelona. Bellaterra. Barcelona. España.

${ }^{6}$ Centro de Transferencia Agroalimentaria. Departamento de Agricultura y Alimentación (CTA). Zaragoza. España.
\end{abstract}

\section{PaLABRAs CLAVE ADICIONALES}

Variabilidad genética. Microsatélites.

\section{RESUMEN}

En este trabajo se analiza la variabilidad genética de la raza autóctona en peligro de extinción Serrana de Teruel, así como su relación con las razas bovinas explotadas en España: Albera, Pajuna, Avileña-Negra Ibérica, Serrana Negra, Pirenaica y Parda de Montaña. La caracterización genética se ha realizado mediante marcadores microsatélites, todos han resultado polimórficos detectándose un total de 198 alelos con una media de 6,79 alelos por locus. Las heterocigosidades observadas y esperadas fueron altas y similares en el equilibrio, con valores de 0,67 y 0,68 respectivamente. A partir del estudio de las relaciones filogenéticas se ha podido observar la cercanía de la raza Serrana de Teruel con las razas de montaña Pirenaica y Parda de Montaña. Mediante el estudio de la estructura genética se observó que el porcentaje de animales correctamente asignados a la Serrana de Teruel para $q \geq 0,8$ fue del $47,5 \%$, apreciándose una clara influencia de la raza Parda de Montaña en los individuos mezclados.

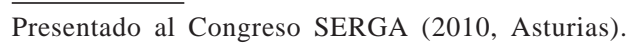

\section{AdDitionAl KEYWORdS}

Genetic diversity. Microsatellites.

\section{SUMMARY}

In this work we analyze by microsatellite markers the genetic diversity, structure and relationships of the indigenous endangered Serrana de Teruel cattle breed with different breeds reared in Spain. All loci were polymorphic and a total of 198 alleles were observed across loci, with a mean of 6.79. Observed and expected heterozygosities values shown the high variability of Serrana de Teruel breed with values of 0.67 and 0.68 respectively. The neighbour net based on Reynolds distances shown the close genetic relationship among Serrana de Teruel and the mountain Parda de Montaña and Pirenaica breeds. STRUCTURE results showed a $47.5 \%$ of correctly assigned individuals to Serrana de Teruel breed using a $\mathrm{q} \geq 0.8$ threshold. The admixed animals shown a clear influence of Parda de Montaña breed.

\section{INTRODUCCIÓN}

La raza bovina autóctona Serrana de Teruel, en peligro de extinción, destaca por 
su rusticidad. Sus aproximadamente 200 ejemplares se distribuyen principalmente por la comarca de Gúdar-Javalambre en las Sierras de Teruel de la Comunidad Autónoma de Aragón, donde está perfectamente adaptada a las duras condiciones climáticas y orográficas. En este trabajo se analiza la variabilidad, estructura y relaciones genéticas de la raza Serrana de Teruel con las razas Albera, Pajuna, Avileña-Negra Ibérica, Serrana Negra, Pirenaica y Parda de Montaña con objeto de conocer su situación actual, para establecer unos esquemas de selección objetivos en función de los intereses productivos que puedan establecerse en las diversas zonas de explotación y que permitan asegurar la preservación de la diversidad genética existente.

\section{MATERIAL Y MÉTODOS}

Se han obtenido muestras de ADN de 80 animales considerados representativos de la raza Serrana de Teruel. Todas las muestras han sido analizadas mediante 30 marcadores microsatélites seleccionados por la FAO para la caracterización de poblaciones bovinas, siguiendo la metodología descrita por Sanz et al. (2007). Además se obtuvieron muestras de ADN para el análisis comparativo de las razas de animales de Albera (80), Pajuna (50), Avileña Negra Ibérica (40), Serrana Negra (52), Pirenaica (50) y Parda de Montaña (50).

Con la información de los distintos genotipos obtenidos de cada animal se obtuvieron las frecuencias alélicas, se analizó el equilibrio Hardy-Weinberg y se calcularon valores de diversidad como son el número

Tabla I. Valores de variabilidad genética en la población Serrana de Teruel utilizando 30 marcadores microsatélites. (Genetic variability values for Serrana de Teruel breed using 30 microsatellites).

\begin{tabular}{ccc}
\hline $\mathrm{He}(\mathrm{SD})$ & $\mathrm{Ho}(\mathrm{SD})$ & FIS \\
$0,68(0,02)$ & $0,67(0,01)$ & 0,039 \\
\hline
\end{tabular}

medio de alelos por locus (A), la tasa de consanguinidad (FIS) y las heterocigosidades observada (Ho) y esperada (He). La relación genética entre poblaciones se analizó mediante el cálculo de la distancia de Reynolds (Reynolds et al., 1983) y se representa mediante la red obtenida con el programa SplitsTree (Huson y Bryant, 2006) utilizando la metodología Neighbor-Net (Bryant y Moulton, 2004).

El estudio de la estructura de la población Serrana de Teruel y la asignación de individuos se llevó a cabo utilizando el programa STRUCTURE (Pritchard et al., 2000). Para cada $K(1 \leq K \leq 9)$ se realizaron 10 repeticiones utilizando un período de quemado de 100000 iteraciones y otras 1000000 en el método MCMC (Monte Carlo de cadenas de Markov) antes de recopilar resultados. Se utilizó el modelo de mezcla de poblaciones con frecuencias alélicas correlacionadas entre poblaciones.

\section{RESULTADOSYDISCUSIÓN}

Todos los microsatélites utilizados han resultado polimórficos. El número de alelos por locus oscila entre 2 (ILSTS005) y 10 (TGLA122), con un número medio de alelos por locus de 6,79. El análisis del equilibio genético Hardy-Weinberg mostró que todos los loci estaban en equilibrio excepto el INRA35, probablemente por la presencia de alelos nulos no detectables.

En general se ha observado una elevada variabilidad y un bajo coeficiente de consanguinidad estimado a partir del valor de FIS (tabla I).

La red obtenida a partir de las distancias genéticas de Reynolds muestra una forma estrellada, apreciándose la relación de la raza Serrana de Teruel con las razas de montaña Parda de Montaña y Pirenaica (Martin-Burriel et al., 2007; Sanz et al., 2007) (figura 1).

El análisis de la estructura genética mostró que el número de poblaciones más probable era 6 (figura 2). Para $K=6$ la pro- 


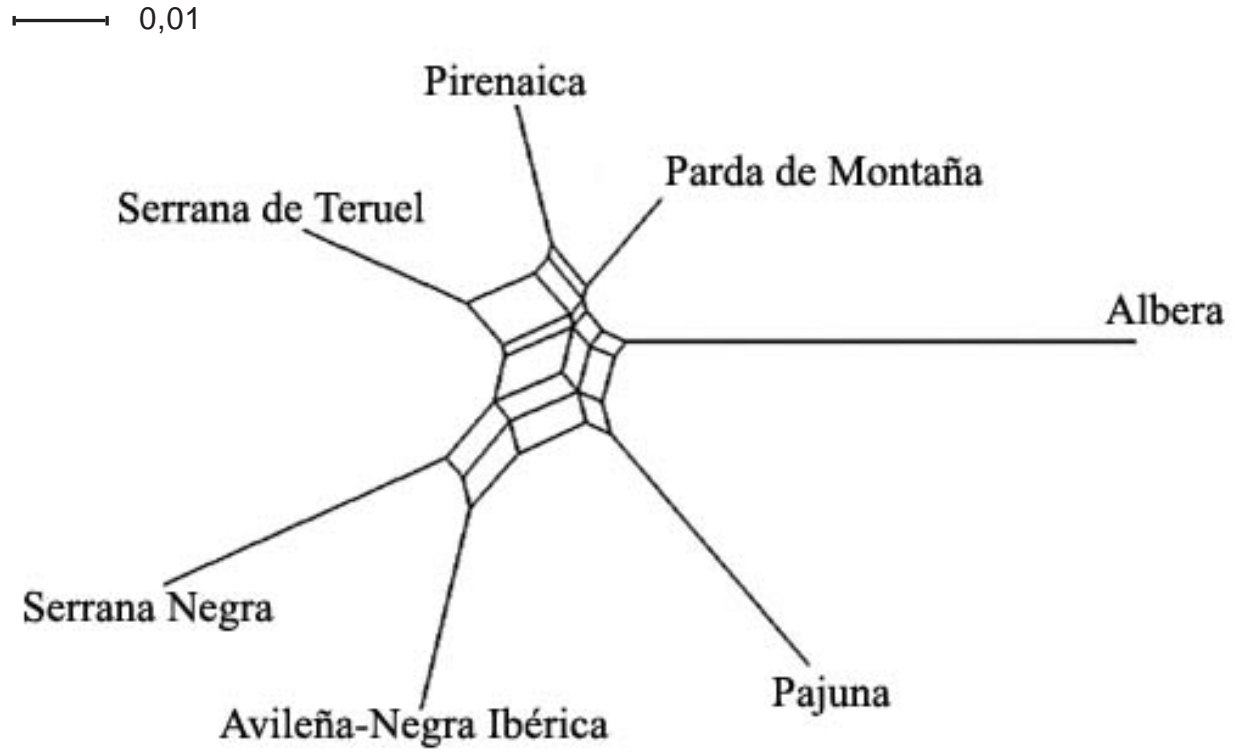

Figura 1. NeighborNet basada en las distancias de Reynolds. (NeighborNet based on Reynolds distances).

porción de individuos asignados correctamente a la Serrana de Teruel fue del $47,5 \%$ para q>0,8 correspondiendo básicamente con los individuos representados por barras más oscuras en la figura 2-B. En el grupo de individuos más heterogéneos se aprecia una clara influencia de la raza Parda de Montaña, lo que concuerda con la información histórica de la raza. Los análisis de asignación son útiles para la identificación de animales representativos del perfil genético de la raza, que pueden ser utilizados en

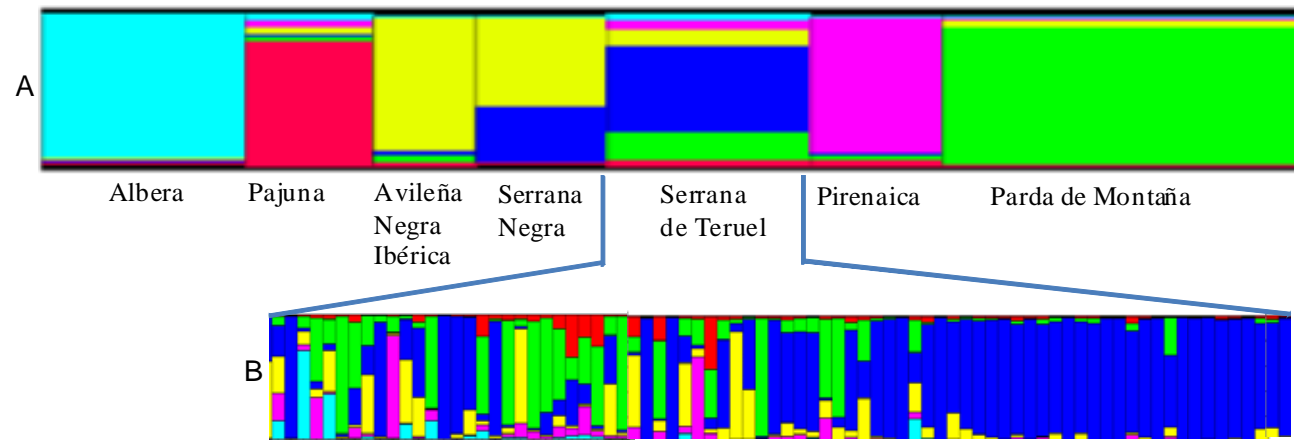

Figura 2. Estructura genética de poblaciones y asignación de individuos de Serrana de Teruel. A: Valores medios de Q por población. B: Valores de q para individuos de Serrana de Teruel. (Genetic structure of 7 Spanish cattle breeds. A: Q values per population. B: Individuals q values). 
programas de selección y conservación de la raza.

\section{CONCLUSIONES}

Los resultados obtenidos indican la riqueza genética de la Serrana de Teruel, diversidad a la que han podido contribuir los cruces conocidos de este bovino con otras poblaciones a lo largo de su historia. Estos resultados pueden contribuir al establecimiento de actuaciones que contribuyan de forma efectiva a preservar y

\section{BIBLIOGRAFÍA}

Bryant, D. and Moulton, V. 2004. Neighbour-net: an agglomerative method for the construction of phylogenetic networks. Mol. Biol. Evol., 21: 255-265.

Huson, D.H. and Bryant, D. 2006. Application of phylogenetic networks in evolutionary studies. Mol. Biol. Evol., 23: 254-267.

Martin-Burriel, I., Rodellar, C., Lenstra, J.A., Sanz, A., Cons, C., Osta, R., Reta, M., De Arguello, S. and Zaragoza, P. 2007. Genetic diversity and relationships of endangered Spanish cattle breeds. J. Hered., 98: 687-691. potenciar la diversidad de la raza y por tanto a su conservación.

\section{AGRADECIMIENTOS}

Financiado con el proyecto PET200705-C03-01 (Caracterización zootécnica, genética y calidad de la canal y de la carne de la población bovina Serrana de Teruel) y RZ2006-00003-C02-02 (Caracterización morfogenética y criopreservación del germoplasma de la Serrana de Teruel, población bovina en peligro de extinción).

Pritchard, J.K., Stephens, M. and Donnelly, P. 2000. Inference of population structure using multilocus genotype data. Genetics, 155: 945959.

Reynolds, J., Weir, B.S. and Cockerham, C.C. 1983. Estimation of the coancestry coefficient: basis for a short-term genetic distance. Genetics, 105: 767-779.

Sanz, A., Martín-Burriel, I., Rodellar, C., Osta, R., Sanz, A., Abril, F. y Zaragoza, P. 2007. Caracterización genética de la población bovina Serrana Negra de Teruel. Arch. Zootec., 56: 461-465.

Archivos de zootecnia vol. 60, núm. 231, p. 372. 